Lernziele

Nach Lektüre dieses Beitrags ...

- haben Sie einen Eindruck über die große Vielfältigkeit, mit der die Lunge bei Tumorerkrankungen beteiligt sein kann,

- kennen Sie die grundsätzlichen Häufigkeiten pulmonaler Tumoren und Metastasen,

- sind Ihnen mögliche Therapieansätze pulmonaler Neoplasien geläufig,

- können Sie die unterschiedlichen Schädigungen der Lunge durch verschiedene Antitumortherapien benennen,

- wissen Sie, wie eine vorbestehende Lungenerkrankung den Verlauf einer Tumorerkrankung beeinflussen kann und wie daher sowohl deren Therapie als auch die einer Tumorerkrankung möglicherweise modifiziert werden müssen.

\title{
Lungenbeteiligung bei Tumorkrankheiten
}

\begin{abstract}
Hintergrund
Die Lunge ist ein zentrales Organ bei Tumorerkrankungen. Neben der Entstehung von Tumoren in der Lunge, den Lungenkarzinomen, und der Metastasierung extrathorakaler Tumoren in die Lungen ist auch ein wechselseitiger Einfluss von Lungenerkrankungen und Tumoren bekannt. Vorbestehende Lungenerkrankungen schränken mögliche Tumortherapieverfahren in teilweise erheblichem $\mathrm{Maß}$ ein und haben prognostische Bedeutung. Schließlich führen unterschiedliche Therapien auch $\mathrm{zu}$ einer konsekutiven Veränderung der Lungenfunktion und können eine Verschlechterung einer vorbestehenden Lungenerkrankung bedingen. In einer Zeit der enormen Weiterentwicklungen systemischer Therapieoptionen bei soliden Tumoren mit erfreulich verbesserter Prognose und sogar deutlichem Anstieg von Langzeitüberlebenden müssen bisherige Strategien zur Be-
\end{abstract}

\section{FIN: BP2101 AT gültig bis 23.02.2021}

Unter Verwendung der Fortbildungs-IdentifikationsNummer (FIN) können Sie für einen begrenzten Zeitraum die Fortbildung kostenfrei nutzen.

Gehen Sie hierzu auf CME.SpringerMedizin.de und geben Sie die FIN in die Suchmaske ein. Sie gelangen direkt zum gewünschten CME-Kurs.

\section{Wissenschaftliche Leitung}

S. Schmitz, Köln

Dieser Beitrag erschien ursprünglich in Der Pneumologe (2020)

17:443-452. https://doi.org/10.1007/s10405-020-00343-4. Die Teilnahme an der zertifizierten Fortbildung ist nur einmal möglich.

best practice onkologie $2021 \cdot 16(1-2): 44-53$

https://doi.org/10.1007/s11654-020-00280-x

Online publiziert: 5. Januar 2021

(c) Springer Medizin Verlag GmbH, ein Teil von Springer Nature 2020 wertung und Therapie zusätzlich bestehender, nichtmaligner Erkrankungen überdacht werden.

Kasuistik. Ein 57-Jähriger Mann leidet an einer COPD (chronische obstruktive Lungenerkrankung) nach GOLD („Global Initiative for Chronic Obstructive Lung Disease“) Grad IIIB und hat vor kurzem seinen über 40 Jahre bestehenden inhalativen Nikotinkonsum eingestellt. Bei einer Krebsvorsorge war er bisher noch nicht, da er beruflich zu beschäftigt gewesen sei. Aufgrund einer subjektiven Änderung seines bekannten morgendlichen Hustens sowie eines starken Drängens auf Abklärung wurde direkt eine CT (Computertomographie) der Lunge durchgeführt, bei der ein 1,4 cm großer peripherer Rundherd im rechten Unterlappen festgestellt wurde.

\section{Maligne und benigne pulmonale Raumforderungen}

\section{Lungenkarzinom}

Das Lungenkarzinom ist die häufigste zum Tode führende Krebsart sowohl bei Männern als auch bei Frauen. Die deutliche Mehrzahl (>80 \%) aller Lungenkarzinome stellen nichtkleinzellige Lungenkarzinome (NSCLC) dar, die ihrerseits in squamöse und nichtsquamösen Formen unterschieden werden. Darüber hinaus gibt es Tumoren, die entweder unterschiedliche histologische Anteile aufweisen oder so undifferenziert sind, dass sie kein eindeutiges morphologisches oder immunhistochemisches Muster aufweisen [1]. Neben histologischen Charakteristika können Lungentumoren auch nach molekularen Veränderungen und stromalen Faktoren (wie Infiltration von spezifischen Leukozyten) weiter differenziert werden. Diese Entwicklung hat die Therapie bereits heute grundlegend verändert und erheblich diversifiziert.

Merke. Das Lungenkarzinom ist eine der häufigsten diagnostizierten Krebsarten und die mit der höchsten Mortalität assoziierte Tumorentität des Menschen. 


\section{Zusammenfassung}

Die Lunge ist bei Tumorerkrankungen sehr oft und auf sehr vielfältige Weise betroffen. Das Lungenkarzinom ist eine der häufigsten Tumorerkrankungen und erfährt gerade in den letzten Jahren eine erhebliche Erweiterung der Therapiemöglichkeiten. Die Lunge ist zudem ein häufiges Metastasierungsorgan verschiedenster Tumorentitäten. Aber auch Therapieansätze wie Tyrosinkinase- und Checkpointinhibitoren, chimäre Antigenrezeptorzelltherapien oder strahlentherapeutische Verfahren können pulmonale Nebenwirkungen verursachen. Schließlich gibt es viele
Patienten, deren pulmonale Grunderkrankung auf den klinischen Verlauf und die Prognose der Tumorerkrankung Einfluss nimmt und ihrerseits beeinflusst werden kann. Beispielhaft werden verschiedene Aspekte wie pulmonale venookklusive Erkrankung, chronisch-obstruktive Atemwegserkrankung und idiopathische pulmonale Fibrose diskutiert.

\section{Schlüsselwörter}

Metastasen · Lungenkarzinom · Pulmonale venookklusive Erkrankung · Chronisch obstruktive Lungenerkrankung · Idiopathische pulmonale Fibrose

\section{Pulmonary involvement in cancers}

\section{Abstract}

The lungs are often involved in tumors and are affected in a wide variety of ways. Lung cancer comprises one of the most common cancer entities and has been characterized by a vast expansion of treatment approaches in recent years. Moreover, the lungs are a common metastatic site of multiple other cancer entities. Various treatment modalities, such as tyrosine kinase inhibitors, checkpoint inhibitors, chimeric antigen receptor cell therapy, and radiotherapy approaches can cause pulmonary side effects.
Finally, many patients suffer from pulmonary comorbidities which may mutually impact the clinical course and prognosis of the cancer disease. As examples, various aspects, such as pulmonary veno-occlusive disease, chronic obstructive pulmonary disease, and idiopathic pulmonary fibrosis are discussed.

Keywords

Neoplasm metastasis · Lung cancer · Pulmonary veno-occlusive disease . Pulmonary disease, chronic obstructive $\cdot$ Idiopathic pulmonary fibrosis
Der Nachweis von Treibermutationen (gehäuft zumeist bei Adenokarzinomen und/oder Nie-Rauchern) bietet die Möglichkeit einer zielgerichteten Therapie, die sich spezifisch gegen die molekulare Alteration richtet. Ausgangspunkt ist das pathophysiologische Verständnis, dass die Treibermutation wesentlich für die Zellfunktionen der Tumorzellen verantwortlich ist. Durch Einsatz sog. zielgerichteter Therapieansätze („targeted therapies“) mit Tyrosinkinaseinhibitoren (TKI) sind häufig langfristige Krankheitskontrollen möglich, bei meist sehr guter Verträglichkeit der (Dauer-)Therapie.

Merke. Alle NSCLC-Patienten im Stadium IV sollten bei Erstdiagnose auf das Vorliegen von therapierbaren molekularen Alterationen untersucht werden [2].

Für Patienten ohne therapierbare Treibermutation galt lange eine alleinige zytotoxische Chemotherapie als Standardbehandlung im metastasierten Stadium, die in einzelnen Fällen über mehrere Linien durchgeführt wurde. Mit der Integration immunmodulatorischer Checkpointinhibitoren veränderten sich das Therapieziel und damit die Standardtherapie vollkommen. Aktuell kann bei einem NSCLC im Stadium IV als Erstlinientherapie - abhängig insbesondere vom histologischen Subtyp, Performancestatus, Komorbiditäten und der Höhe der PD-L1Expression (PD-L1: „programmed cell death 1 ligand 1“) - zwischen

- einer Monotherapie mit einem Checkpointinhibitor,

- einer Kombinationschemotherapie,

- einer Kombinationschemotherapie mit einem Checkpointinhibitor oder
- einer Kombinationschemotherapie mit einem Checkpointinhibitor und einem Anti-VEGF-Antikörper (VEGF: „vascular endothelial growth factor“ [vaskulärer endothelialer Wachstumsfaktor])

ausgewählt werden [3]. In Entwicklung sind zudem Kombinationen verschiedener Checkpointinhibitoren und einer Chemotherapie. Da ein Teil der Patienten in eine länger andauernde Krankheitskontrolle gebracht werden kann, ändert sich das bisher dominant formulierte Therapieziel des Erhalts und der Verbesserung der Lebensqualität des Patienten. Zudem ergeben sich neue Fragen wie erforderliche Immuntherapiedauer oder Möglichkeiten der Reexposition mit Checkpointinhibitoren nach Progress.

Schließlich werden trotz Stadium IV häufiger lokale Therapieverfahren wichtig. Beispielweise ist in der aktuellen TNMKlassifikation (T: Tumorstadium, N: Lymphknotenstadium, M: Metastasierung) ein oligometastasiertes Tumorstadium definiert, bei dem lokale Therapieverfahren interdisziplinär diskutiert werden sollten. In einer retrospektiven US-amerikanischen Registerstudie mit knapp 35.000 Patienten mit bis zu 4 verschiedenen Metastasenlokalisationen profitierten Menschen, bei denen zusätzlich zu einer systemischen eine lokale Therapie, insbesondere eine operative Resektion, durchgeführt wurde, hinsichtlich des Gesamtüberlebens [4].

Merke. In den letzten Jahren haben sich die therapeutischen Optionen zur Behandlung des Lungenkarzinoms erheblich erweitert und insbesondere in den lokal fortgeschrittenen und metastierten Stadien des NSCLC die bisherige Standardtherapie revolutioniert. 
In frühen Erkrankungsstadien (Stadium I-III) steht der Versuch der Kuration im Vordergrund, sodass lokale Therapieverfahren wie chirurgische Entfernung und strahlentherapeutische Verfahren interdisziplinär diskutiert werden müssen. Aufgrund der frühen Metastasierungstendenz werden auch systemische Therapien wie Chemotherapie und - aktuell nur im Stadium nach Chemo- und Strahlentherapie zugelassen - Checkpointinhibitoren zur Verbesserung der Heilungschance eingesetzt.

Kasuistik. Eine PET-CT (PET: Positronenemissionstomographie) zeigte eine FDG-Anreicherung (FDG: Fluordesoxyglukose). In einer CT-gesteuerten Punktion ließ sich ein TTF1-positives (TTF1: thyroidaler Transkriptionsfaktor 1) Adenokarzinom nachweisen. Aufgrund der schlechten Lungenfunktion wurde eine stereotaktische Bestrahlung durchgeführt.

Das kleinzellige Lungenkarzinom stellt eine besonders aggressive Tumorentität dar und ist durch schnellen Progress, sehr frühe Metastasierung und schlechte Prognose charakterisiert. Nur wenige Patienten werden in einem frühen Erkrankungsstadium diagnostiziert, bei dem in ausgewählten Fällen eine chirurgische Resektion diskutiert werden kann. Zumeist wird bei nicht hämatogen-metastasierten Stadium eine Radiochemotherapie durchgeführt, mit prophylaktischer Ganzhirnbestrahlung. Bei Patienten im Stadium IV ist eine Kombination zwischen platinhaltiger Chemotherapie und einem PD-L1-Inhibitor (derzeit nur Atezolizumab zugelassen) neuer Therapiestandard.

Neben den sehr häufig diagnostizierten Karzinomen kommen auch eine Reihe mesenchymaler und weiterer Tumoren vor. Die Diagnose und Therapie von Lungentumoren sind im Detail an anderer Stelle ausführlicher beschrieben $[3,5,6]$.

Kasuistik.. Nach 2 Jahren traten bei dem Patienten mehrere Lymphknotenvergrößerungen mediastinal sowie Leberrundherde auf. In einer erneuten Biopsie wurde ein Rezidiv des vorbekannten NSCLC nachgewiesen. Aufgrund einer hohen PD-L1Expression und dem Fehlen von Treibermutationen wurde eine Checkpointinhibitortherapie eingeleitet. Hierunter kam es zu einer partiellen Remission.

\section{Pulmonale Rundherde}

Pulmonale Herde werden in Abhängigkeit vom Patientenkollektiv recht häufig detektiert, die Mehrzahl davon ohne begleitende klinische Symptome. Asymptomatische, solitäre Rundherde haben eine breite Differenzialdiagnose, die neben einem Malignom auch gutartige Tumoren wie Hamartome und benigne fibröse Tumoren sowie eine Reihe gutartiger Veränderungen wie Tuberkulose, Sarkoidose und Silikose umfassen. Allgemein können neben der Tumorgröße auch die Größenänderung beim Vergleich mit früheren Aufnahmen bzw. im Verlauf, das radiologische Erscheinungsbild und eine Anreicherung mit radioaktiv markierter Glukose $\left({ }^{18} \mathrm{~F}\right.$-Fluordesoxyglukose; $\left.{ }^{18} \mathrm{~F}-\mathrm{FDG}\right)$ im PET zur Abschätzung der Wahrscheinlichkeit einer malignen Diagnose hinzugezogen werden. Verschiedene Gesellschaften wie die „Fleischner Society“ und das „American College of Chest Physicians“ (ACCP) definierten Kriterien für die weitere Abklärung bzw. Beobachtungsintervalle [7, 8].

\section{Lungenmetastasen}

Sie werden häufig bei fortgeschrittener Tumorerkrankung unterschiedlicher solider Tumoren gefunden einschließlich Tumoren von Kolon, Rektum, Niere, Mamma, Prostata und Oropharynx. Darüber hinaus kommt es zu einer die Lungen präferierenden Metastasierung beim Chorionkarzinom, Osteosarkom, Weichteilsarkom, Hodentumor, Ewing-Sarkom und Schilddrüsenkarzinom. Da die Diagnose pulmonaler Metastasen in allen Fällen eine systemische Erkrankung anzeigt, die im ganz überwiegenden Fall eine palliative Therapiesituation darstellt, sollte prinzipiell eine systemische Therapie diskutiert werden [5].

Allerdings zeigen die Tumoren im metastasierten Stadium sehr unterschiedliche Verläufe, die sich beispielsweise in der Definition oligometastatischer Krankheitsstadien widerspiegeln. Daher sollte bei limitierter Metastasenanzahl in Abhängigkeit der individuellen Situation im Einzelfall auch eine lokale Therapie diskutiert werden [5]. Hierbei müssen die zugrunde liegende Tumorentität, der Performancestatus des Patienten, der individuelle Krankheitsverlauf, mögliche therapeutische Alternativen und natürlich der Patientenwunsch berücksichtigt werden. Bei unklaren Befunden ist zudem die histologische Sicherung wichtig, um ein Zweitkarzinom wie z. B. ein primäres Lungenkarzinom auszuschließen. Prinzipiell sollte die Indikation zur pulmonalen Metastasenresektion unter kurativer Zielsetzung im interdisziplinären Dialog zwischen Thoraxchirurgen, Onkologen, Strahlentherapeuten und Organspezialisten gestellt werden.

Merke. Bei Lungenmetastasen von extrathorakalen Tumoren können bei einigen Patienten auch lokale Therapieverfahren nach interdisziplinärer Diskussion eingesetzt werden.

\section{Pulmonale Toxizität antineoplastischer Substanzen}

Systemische Therapieverfahren haben sich in den letzten Jahren erheblich erweitert. Prinzipiell können alle Medikamente verschiedene pulmonale Nebenwirkungen verursachen, die oft unspezifisch sind. Bekannte pneumotoxische Nebenwirkungen können für eine Vielzahl von Medikamenten auf der Webseite pneumotox.com eingesehen werden. Zudem sind die klinische Präsentation von Lungenveränderungen durch Krebstherapeutika sowie deren mögliche Auswirkungen auf die pulmonale Funktion sehr variabel und daher eine differenzialdiagnostische Herausforderung [9]. Beispielsweise muss neben einer Arzneimittelnebenwirkung bei Fieber und radiologischen Infiltraten auch an pulmonale Infekte, eine pulmonale Progression der malignen Grunderkrankung (insbesondere Lymphangiosis carcinomatosa), Lungenembolien und die Exazerbation einer bereits bestehenden, tumorunabhängigen Lungenerkrankung gedacht werden.

Merke. Bekannte pulmotoxische Nebenwirkungen können für eine Vielzahl von Medikamenten auf der Webseite pneumotox. com eingesehen oder auch selbst gemeldet werden.

Beispiele für antineoplastische Substanzen, bei denen eine pulmonale Toxizität bekannt ist, sind Bleomycin und Methotrexat (MTX). Bleomycin, das v. a. zur Therapie von Lymphomen und Keimzelltumoren eingesetzt wird, ist in Bezug auf eine Lungenschädigung eines der am besten charakterisierten Medikamente. 
Die Inzidenz einer Pulmotoxizität ist dosisabhängig und reicht von 0-46 \% je nach Patientenkollektiv und Kovariablen mit einer Letalität bis zu $3 \%$. Zumeist tritt eine interstitielle Lungenerkrankung (ILD) während, teilweise aber auch bis zu wenigen Jahren nach Therapie auf [10]. Auch für Methotrexat wurde bisher eine Pneumonitis als relevante Nebenwirkung diskutiert, und besonders im Rahmen rheumatologischer Erkrankungen mit interstitieller Lungenkrankheit wurde oft das Absetzen von MTX empfohlen. Dieses Vorgehen wird aufgrund der aktuellen Daten in Frage gestellt. Eine Abhängigkeit zur Dosis oder Therapiedauer besteht allerdings nicht. Bei Verdacht auf eine MTX-induzierte Pneumonitis sollten die MTX-Gabe abgebrochen und eine Behandlung mit systemischen Glukokortikoiden eingeleitet werden.

\section{Pulmonale venookklusive Erkrankung bei Alkylanzien oder Mitomycin}

Eine seltene pulmonale Nebenwirkung ist die Entwicklung einer pulmonalen venookklusiven Erkrankung (PVOD, [11]). Diese stellt eine seltene Ursache der pulmonalen Hypertonie dar und ist v. a. nach Behandlung mit Alkylanzien (insbesondere Cyclophosphamid) oder Mitomycin C beschrieben. Sie ist durch eine fibröse Proliferation der Intima der kleinen Venen und Venolen charakterisiert und häufig mit pulmonalkapillarer Dilatation und Proliferation assoziiert, die zu einer präkapillaren pulmonalen Hypertonie führen. Computertomographisch stellen betonte septale Linien, eine zentroazinäre Milchglastrübung und eine mediastinale Lymphadenopathie typische Befunde einer PVOD dar. Diese ist mit einer schlechten Prognose assoziiert. Die Therapie mit den bei der pulmonal arteriellen Hypertonie (PAH) zugelassenen Medikamenten ist mit dem Risiko der Entwicklung eines Lungenödems verbunden.

Merke. Eine pulmonale venookklusive Erkrankung ist eine seltene Nebenwirkung insbesondere nach Behandlung mit Alkylanzien oder Mitomycin C.

\section{Tyrosinkinaseinhibitoren}

Die meisten pulmonalen Toxizitäten von TKI haben eine geringe Ausprägung und sind bei Dosisreduktion oder Therapiepause vollständig reversibel [12]. Dennoch können eine frühe Diagnose und im Bedarfsfall medikamentöse Intervention mit systemischen Steroiden den Verlauf einer Pneumonitis deutlich verbessern.

\section{Checkpointinhibitoren}

Immuncheckpointinhibitoren werden aufgrund ihrer klinischen Wirksamkeit breit eingesetzt. Die immunstimulierenden Effekte bedingen jedoch die Induktion von Autoimmunphänomenen, die auch pulmonale Nebenwirkungen wie ILD, lymphozytäre Pneumonien und sarkoidoseähnliche granulomatöse Lungenerkrankungen einschließen. Für PD-1-Inhibitoren (PD-1: „programmed death protein 1 “) wurden pulmonale Nebenwirkungen bei bis zu $13 \%$ der Behandelten berichtet, wobei nur ungefähr 2-3 \% als höhergradig klassifiziert wurden [13]. Das Nebenwirkungsspektrum von PD-L1-Inhibitoren ist dem von PD-1-Inhibitoren vergleichbar.

Die Pneumonitis ist die häufigste zum Therapieabbruch führende Komplikation, die einen sehr variablen Zeitpunkt des
Beginns und verschiedene klinische, radiologische und pathologische Formen zeigen kann. Die klinischen Symptome sind uncharakteristisch, wie trockener, unproduktiver Husten, Tachy- und Dyspnoe, Tachykardien und Fatigue. Das CT-Bild ist ebenfalls heterogen und kann Milchglasinfiltrate und Charakteristika einer organisierenden oder einer interstitiellen Pneumonie oder einer Hypersensitivitätspneumonitis zeigen [14]. Die Diffusionskapazität ist früh vermindert. Abhängig vom Schweregrad muss - entsprechend verschiedener veröffentlichter Therapieempfehlungen - neben einer Therapiepausierung oder -terminierung auch die Einleitung einer Steroidbehandlung diskutiert werden [14].

Eine Erhöhung des Pneumonierisikos ist bei Checkpointinhibitoren nicht eindeutig belegt und von pulmonalen Vorerkrankungen und dem verwendeten Checkpointinhibitor abhängig [13]. Ein möglicher Einfluss auf den Verlauf einer Infektion mit COVID-19 ist aktuell noch nicht beschrieben.

Merke. Eine Pneumonitis kann sich bei verschiedenen Medikamenten als Nebenwirkung entwickeln und sollte leitliniengerecht diagnostiziert und behandelt werden [14].

Kasuistik. Nach 3 Monaten Therapie klagte der Patient über eine Verschlechterung der Luftnot. Im CT des Thorax zeigten sich interstitielle Infiltrate, die auf eine Pneumonitis hinwiesen. Nach Abbruch der Therapie und Einleitung einer Steroidbehandlung mit $2 \mathrm{mg} / \mathrm{kg}$ Körpergewicht kam es rasch zu einer Verbesserung der Symptome und einem Rückgang der Infiltrate.

\section{Therapie mit chimären Antigenrezeptor-T-Zellen (CART)}

Eine Variante der aktiven Immuntherapie ist die Verwendung von chimären Antigenrezeptor-T-Zellen (CART), bei der T-Zellen des Patienten modifiziert werden, um die Lokalisation der Tumorzelle und die Aktivierung und Proliferation der T-Zellen zu verbessern. Nach ersten, beeindruckenden Erfolgen insbesondere bei Kindern mit refraktärer Leukämie und Erwachsenen mit M. Hodgkin wird dieser Ansatz auch bei anderen Entitäten untersucht. Eine Herausforderung dabei ist die Kontrolle einer immunvermittelnden (Über-)Reaktion [15]. Die CART-Therapie ist assoziiert mit akuter, manchmal sehr rasch progredienter Toxizität wie dem CRS („cytokine release syndrome“), Neurotoxizität, Zytopenien und Tumorlysesyndrom, die dem behandelnden Arzt bekannt sein müssen. Das CRS, auch als Zytokinsturm bezeichnet, ist eine systemische, inflammatorische Antwort, welche durch freigesetzte Zytokine der CART und anderen beteiligten Immunzellen hervorgerufen wird und - seltener - auch bei Therapien mit Checkpointinhibitoren oder anderen Antikörpern (z. B. Blinatumomab) auftreten kann. Es ist charakterisiert durch Fieber, Hypoxie, Tachykardie, Hypotension und Multiorganversagen. In einem Konsensusstatement der ASTCT („American Society for Transplantation and Cellular Therapy") wird eine Graduierung des CRS empfohlen; zudem wurden Therapieempfehlungen formuliert $[16,17]$. Therapieansätze schließen Sauerstoffgabe, mechanische Ventilation, Anti-Interleukin-6-Antikörper und Steroide ein.

Merke. Bei CART-Therapien ist das „cytokine release syndrome“ eine schwere, akute Nebenwirkung. 


\section{Strahlenpneumonitis}

Eine konventionell fraktionierte thorakale Bestrahlung kann eine akute oder chronische Schädigung verschiedener Zellen, v. a. Endothelzellen und Typ-I- und -II-Pneumozyten, sowie eine Zytokindisbalance und Lymphozyteninfiltration des Lungenparenchyms verursachen, die klinisch in einer Kombination von Alveolitis und interstitieller Entzündung und später in einer postradiogenen Lungenfibrose resultieren. Eine Pneumonitis nach Strahlen- oder Strahlenchemotherapie tritt zumeist innerhalb der ersten 6 Monate auf, und die Inzidenz wird mit bis zu $16 \%$ aller Patienten beschrieben [18]. Sie hängt von der kumulativ verabreichten Strahlendosis ab [19].

Die Symptome sind zumeist unspezifisch, wie trockener Husten, Atemnot und in manchen Fällen auch leichte Körpertemperaturerhöhung. Charakteristisch ist ein Diffusionskapazitätsabfall, der auch bei unveränderter Einsekundenkapazität (FEV1) und nicht eingeschränkter Lungenkapazität vorhanden sein kann. Feingeweblich findet sich dabei typischerweise das Bild einer interstitiellen Pneumonie.

Merke. Eine Strahlenpneumonitis ist ein häufiges Phänomen nach stattgefundener thorakaler Radiatio.

\section{Knochenmarktransplantation bei malignen hämatolo- gischen Krankheiten}

Eine hämatopoetische Stammzellentransplantation (HSCT) kann mit frühen oder späten schweren Toxizitäten assoziiert sein. Im Rahmen einer HSCT können viele Komplikationen auftreten, die beispielsweise durch die Konditionierung, die Zytopenie, die durch die nachfolgende Immunsuppression noch verstärkt wird, Endothelzellschäden oder das Auftreten einer Graft-versus-Host-Erkrankung (GvhD) verursacht werden. Infektionen gehören zu den häufigsten Todesursachen nach HSCT. Darüber hinaus definierte das NIH („National Institutes of Health") ein idiopathisches Pneumoniesyndrom (IPS) als alveolaren Schaden nach erfolgter HSCT bei Fehlen einer aktiven Infektion der unteren Atemwege oder anderer kausaler, insbesondere kardialer, Ursachen [20]. Das IPS tritt bei ungefähr 2-8 \% aller Patienten innerhalb von 12 Tagen nach allogener HSCT auf und führt bei über $60 \%$ der Betroffenen zum Tode [20]. Die klinische Präsentation ist äußerst vielfältig und wird zeitlich unterschiedlich beobachtet:

- Prä-Engraftment-Phase (z. B. „transfusion-related lung injury") 1-4 Wochen nach der Transplantation,

- frühe Post-Engraftment-Phase (bis zu Tag 100) und

- späte Phase (nach Tag 100).

Zudem können die einzelnen Krankheitsbilder auch aufgrund der primären anatomischen Schädigung abgegrenzt werden: Schädigungen

- des Lungenparenchyms (z. B. akute interstitielle Pneumonitis, Strahlenpneumonitis, eosinophile Pneumonie),

- des Gefäßendothels (z. B. diffuse alveoläre Hämorrhagie, pulmonale venookklusive Erkrankung) oder

- des Alveolarepithels (z. B. kryptogen organisierende Pneumonie [COP], Bronchiolitis obliterans).

Bei raschen Verläufen resultiert häufig ein Multiorganversagen. Pulmonale Spätschäden manifestieren sich insbesondere als Bronchiolitis obliterans (BO), COP oder Lungenfibrose.
Merke. Eine hämatopoetische Stammzellentransplantation kann auf vielfältige Weise und auch zeitlich sehr variabel zu pulmonalen Schädigungen führen.

\section{Einflüsse auf vorbestehende pulmonale Erkrankungen}

Die gegenseitigen Wechselwirkungen zwischen einer onkologischen Erkrankung und einer vorbestehenden pulmonalen Komorbidität sind noch unzureichend erforscht. Zumeist wurden in der Vergangenheit die Prognosen der jeweiligen Erkrankungen gegeneinander abgewogen und anschließend die Erkrankung mit der schlechteren Prognose bevorzugt betrachtet. In den letzten Jahren wurde jedoch zunehmend die Heterogenität von Diagnostik, Therapie und Verlauf einer onkologischen Erkrankung evident. Vorbestehende, chronische, Krankheiten wie pulmonale, neurologische, kardiale, rheumatische und psychiatrische Erkrankungen, können den Diagnosezeitpunkt und damit auch das Tumorstadium bei Diagnosestellung sowie die therapeutischen Möglichkeiten und Behandlungseffekte beeinflussen [21]. Eine rheumatische Erkrankung kann beispielsweise die Durchführung einer Checkpointinhibitortherapie erschweren oder gar unmöglich machen. Verschiedene zytotoxische Substanzen beeinflussen die kardiale Funktion und können langfristig eine vorbestehende Herzinsuffizienz verschlechtern. Aufgrund der zunehmend besseren Prognose zumindest bei einem Teil unserer Patienten rücken diese Fragen mehr und mehr in den Vordergrund und müssen in zukünftigen Studien adressiert werden.

Merke. Vorbestehende pulmonale Erkrankungen können den Zeitpunkt der Diagnose verzögern und damit auch das Tumorstadium zum Diagnosezeitpunkt sowie die therapeutischen Möglichkeiten und Wirkungen beeinflussen.

Es wurden auch Gemeinsamkeiten unterschiedlicher Erkrankungen festgestellt: Beispielsweise gibt es pathophysiologische Charakteristika bei der pulmonal arteriellen Hypertonie (PAH) wie Induktion von Proliferation, Antiapoptose und Angiogenese, metabolische Dysregulation und proinflammatorische Stimuli, die auch bei malignen Tumoren beobachtet werden [22]. Auch wenn pulmonale Epithelzellen bei der PAH nicht maligne entarten, ist es doch nachvollziehbar, dass Antitumortherapien auch direkt vorbestehende Erkrankungen wie die PAH beeinflussen können.

Für die chronisch obstruktive Atemwegserkrankung (COPD) ist seit längerem ein Zusammenhang mit der Entwicklung und dem Verlauf einer Lungenkrebserkrankung bekannt [23, 24]. Einige, aber nicht alle Studien legen eine Unabhängigkeit dieses Risikofaktors vom Nikotinkonsum nahe [25]. Auch gilt die bestehende Diagnose einer COPD mit oder ohne Emphysem als ungünstiger prognostischer Faktor eines Lungenkarzinoms [26]. Dies könnte zumindest teilweise durch ähnliche stromale oder zelluläre Veränderungen wie proinflammatorische Zytokine, erhöhte Expression von Matrixmetalloproteinasen, Aktivierung von VEGFR1 („vascular endothelial growth factor receptor 1“), verkürzte Telomere oder veränderte Zellsteuerung (z. B. Aktivierung von NF- $\mathrm{KB}$ [,nuclear factor kappa light chain enhancer of activated B-cells"]) begründet sein [23].

Schließlich wurden auch zahlreiche Zusammenhänge zwischen idiopathischer pulmonaler Fibrose (IPF) und Lungenkarzinomen beschrieben. Die IPF ist als Risikofaktor für die Ent- 
wicklung eines solchen eindeutig erkannt [27]. Auch hier sind zahlreiche pathophysiologische Gemeinsamkeiten beschrieben. Manche Therapien wie antiangiogene bzw. antistromale TKI (z. B. Nintedanib) sind sowohl bei der IPF als auch beim Lungenkarzinom wirksam. Darüber hinaus kann bei Therapie eines Lungenkarzinoms eine akute Exazerbation (AE) einer vorbestehenden ILD ausgelöst werden, die ihrerseits mit deutlich erhöhter Mortalität assoziiert ist. So ist das Risiko einer AE durch operative Eingriffe deutlich erhöht [28]. Aus verschiedenen Fallberichten gibt es Hinweise, dass auch nichtpulmonale Operationen eine AE der IPF auslösen können [9, 27]. Möglicherweise sind intraoperativ hohe Sauerstoffkonzentrationen, hohe Atemwegsdrücke und -volumina wichtige auslösende Faktoren. Auch eine thorakale Radiatio ist als Risikofaktor für eine AE der IPF bekannt. Darüber hinaus können auch fast alle Chemotherapeutika zu einer AE der IPF bzw. einer Verschlechterung der ILD führen [9].

Merke. Für zahlreiche pulmonale Erkrankungen gibt es mit Tumorerkrankungen wechselseitige Einflüsse, die bisher noch ungenügend verstanden sind.

\section{Ausblick}

Die beschriebenen Entwicklungen spiegeln die große Dynamik der Entwicklung der Tumortherapien und der komplizierten pulmonalen Interaktionen wider. Die Testung bestimmter Marker nicht nur in der Primärdiagnostik, sondern auch während der Therapie oder bei Krankheitsprogression wird zunehmend sinnvoll und notwendig, was eine weitere klinische Herausforderung darstellen kann. Insbesondere die Fragen der Überwachung, Therapie und Berücksichtigung der Prognose bei pulmonalen Komorbiditäten stellen aktuelle Herausforderungen dar, die in klinischen Studien adressiert werden sollten.

\section{Fazit für die Praxis}

- Die Lunge kann auf unterschiedliche Weise bei Tumorerkrankungen beteiligt sein - entweder als Ort der Tumorlokalisation, als möglicher Ort von Nebenwirkungen der Tumortherapien oder durch wechselseitige Beeinflussung von Tumor- und vorbestehender Lungenerkrankung.

- Die Lunge ist Lokalisation der weltweit am häufigsten zum Tode führenden Tumorentität sowie ein häufiger Ort metastatischer Absiedlungen extrathorakaler Tumoren.

- Zahlreiche und unterschiedliche Therapieansätze haben pulmonale Nebenwirkungen, wobei dem behandelnden Arzt insbesondere das Risiko und die mögliche Therapie einer Pneumonitis bekannt sein müssen.

- Viele Patienten leiden an pulmonalen Vorerkrankungen, die durch die Tumorerkrankung beeinflusst werden und auf diese selbst Auswirkungen haben können. Zudem gibt es zahlreiche gemeinsame pathophysiologische Mechanismen.

Literatur

1. Travis WD, Brambilla E, Riely GJ (2013) New pathologic classification of lung cancer: relevance for clinical practice and clinical trials. J Clin Oncol 31:992-1001

2. Griesinger F, Eberhardt W, Früh M et al (2019) Lungenkarzinom, nicht-kleinzellig (NSCLC). Onkopedia. https://www.onkopedia.com/de/onkopedia/guidelines/ lungenkarzinom-nicht-kleinzellig-nsclc/@@guideline/html/index.html
3. Leitlinienprogramm Onkologie (Deutsche Krebsgesellschaft DK, Awmf) (2018) Prävention, Diagnostik, Therapie und Nachsorge des Lungenkarzinoms

4. Uhlig J, Case MD, Blasberg JD et al (2019) Comparison of survival rates after a combination of local treatment and systemic therapy vs systemic therapy alone for treatment of stage IV non-small cell lung cancer. JAMA Netw Open 2:e199702

5. Reinmuth N, Lindner M (2017) Besonderheiten in der pneumologischen Onkologie. Der Pneumologe 6

6. Reinmuth N, Schumann C (2018) Fundamental changes in the treatment of lung cancer. Pneumologie 72:732-735

7. Gould MK, Donington J, Lynch WR et al (2013) Evaluation of individuals with pulmonary nodules: when is it lung cancer? Diagnosis and management of lung cancer, 3rd ed: American College of Chest Physicians evidence-based clinical practice guidelines. Chest 143:e93S-e120S

8. Macmahon H, Naidich DP, Goo JM et al (2017) Guidelines for management of incidental pulmonary nodules detected on CT images: from the Fleischner society 2017. Radiology. https://doi.org/10.1148/radiol.2017161659

9. Kreuter M, Ehlers-Tenenbaum S, Schaaf M et al (2015) Treatment and outcome of lung cancer in idiopathic interstitial pneumonias. Sarcoidosis Vasc Diffuse Lung Dis 31:266-274

10. Rosenow EC 3rd (1994) Drug-induced pulmonary disease. Dis Mon 40:253-310

11. Ranchoux B, Gunther S, Quarck R et al (2015) Chemotherapy-induced pulmonary hypertension: role of alkylating agents. Am J Pathol 185:356-371

12. Barber NA, Ganti AK (2011) Pulmonary toxicities from targeted therapies: a review. Target Oncol 6:235-243

13. Su Q, Zhu EC, Wu JB et al (2019) Risk of pneumonitis and pneumonia associated with immune checkpoint inhibitors for solid tumors: a systematic review and meta-analysis. Front Immunol 10:108

14. Haanen J, Carbonnel F, Robert C et al (2018) Management of toxicities from immunotherapy: ESMO Clinical Practice Guidelines for diagnosis, treatment and follow-up. Ann Oncol 29:iv264-iv266

15. Wall DA, Krueger J (2020) Chimeric antigen receptor T cell therapy comes to clinical practice. Curr Oncol 27:S115-S123

16. Gutierrez C, Mcevoy C, Mead E et al (2018) Management of the critically ill adult chimeric antigen receptor-T cell therapy patient: a critical care perspective. Crit Care Med 46:1402-1410

17. Lee DW, Santomasso BD, Locke FL et al (2019) ASTCT consensus grading for cytokine release syndrome and neurologic toxicity associated with immune effector cells. Biol Blood Marrow Transplant 25:625-638

18. Hanania AN, Mainwaring W, Ghebre YT et al (2019) Radiation-induced lung injury: assessment and management. Chest 156:150-162

19. Monson JM, Stark P, Reilly JJ et al (1998) Clinical radiation pneumonitis and radiographic changes after thoracic radiation therapy for lung carcinoma. Cancer 82:842-850

20. Panoskaltsis-Mortari A, Griese M, Madtes DK et al (2011) An official American Thoracic Society research statement: noninfectious lung injury after hematopoietic stem cell transplantation: idiopathic pneumonia syndrome. Am J Respir Crit Care Med 183:1262-1279

21. Renzi C, Kaushal A, Emery J et al (2019) Comorbid chronic diseases and cancer diagnosis: disease-specific effects and underlying mechanisms. Nat Rev Clin Oncol 16:746-761

22. Cool CD, Kuebler WM, Bogaard HJ et al (2020) The hallmarks of severe pulmonary arterial hypertension: the cancer hypothesis - ten years later. Am J Physiol Lung Cell Mol Physiol 318(6):L1115-L1130

23. Seijo LM, Zulueta JJ (2017) Understanding the links between lung cancer, COPD, and emphysema: a key to more effective treatment and screening. Oncology 31:93-102

24. Wasswa-Kintu S, Gan WQ, Man SF et al (2005) Relationship between reduced forced expiratory volume in one second and the risk of lung cancer: a systematic review and meta-analysis. Thorax 60:570-575 
25. Wilson DO, Weissfeld JL, Balkan A et al (2008) Association of radiographic emphysema and airflow obstruction with lung cancer. Am J Respir Crit Care Med 178:738-744

26. Gillissen A, Haidl P, Khlhaufl M et al (2016) The pharmacological treatment of chronic obstructive pulmonary disease. Dtsch Arztebl Int 113:311-316

27. Kinoshita T, Goto T (2019) Molecular mechanisms of pulmonary fibrogenesis and its progression to lung cancer: a review. Int J Mol Sci 20(6):1461

28. Juarez MM, Chan AL, Norris AG et al (2015) Acute exacerbation of idiopathic pulmonary fibrosis - a review of current and novel pharmacotherapies. J Thorac Dis 7:499-519

\section{Korrespondenzadresse}

\section{PD Dr. N. Reinmuth}

Abteilung für Thorakale Onkologie, Asklepios Fachkliniken München-Gauting Robert-Koch-Allee 2, 82131 Gauting, Deutschland n.reinmuth@asklepios.com

\section{Einhaltung ethischer Richtlinien}

Interessenkonflikt. Gemäß den Richtlinien des Springer Medizin Verlags werden Autoren und Wissenschaftliche Leitung im Rahmen der Manuskripterstellung und Manuskriptfreigabe aufgefordert, eine vollständige Erklärung zu ihren finanziellen und nichtfinanziellen Interessen abzugeben.

Autoren. N. Reinmuth: Finanzielle Interessen: Referentenhonorar: Roche, MSD, BMS, Boehringer-Ingelheim, AstraZeneca, Pfizer, Takeda. Bezahlter Berater/interner Schulungsreferent/Gehaltsempfänger o. ä.: AbbVie, Roche, MSD, Merck, BMS, Boehringer-Ingelheim, AstraZeneca, Pfizer, Takeda. - B. Nichtfinanzielle Interessen: Chefarzt, Thorakale Onkologie, Asklepios Fachkliniken München-Gauting. S.-C. Mavi: A. Finanzielle Interessen: S.-C. Mavi gibt an, dass kein finanzieller Interessenkonflikt besteht. - B. Nichtfinanzielle Interessen: Fachärztin für Pneumologie und Innere Medizin, Oberärztin an der Asklepios Fachklinik München-Gauting, Pneumologie; Mitglied in der DGP (Deutsche Gesellschaft für Pneumologie).

Wissenschaftliche Leitung. Die vollständige Erklärung zum Interessenkonflikt der Wissenschaftlichen Leitung finden Sie am Kurs der zertifizierten Fortbildung auf www.springermedizin.de/cme.

Der Verlag. erklärt, dass für die Publikation dieser CME-Fortbildung keine Sponsorengelder an den Verlag fließen.

Für diesen Beitrag wurden von den Autoren keine Studien an Menschen oder Tieren durchgeführt. Für die aufgeführten Studien gelten die jeweils dort angegebenen ethischen Richtlinien. 


\section{CME-Fragebogen}

FIN BP2101AT gültig bis 23.02.2021

Dieser CME-Kurs ist zwölf Monate auf CME.SpringerMedizin. de verfügbar. Sie finden ihn am schnellsten, wenn Sie die oben genannte FIN oder den Titel des Beitrags in das Suchfeld eingeben. Alternativ können Sie auch mit der Option „Kurse nach Zeitschriften" zum Ziel navigieren.
- als e.Med-Abonnent von springermedizin.de

- als registrierter Abonnent dieser Fachzeitschrift

- zeitlich begrenzt unter Verwendung der abgedruckten FIN.
Teilnehmen und Punkte sammeln können Sie
? Was ist das Hauptsymptom der strahlenbedingten Pneumonitis?

Atemabhängige Thoraxschmerzen

Trockener Husten und Atemnot

Hohes Fieber

Hämoptyse

Thorakale Schmerzen

? Was ist der häufigste Primärtumor bei nachgewiesenem Tumorbefall der Lunge?

Osteosarkom

Primäres nichtkleinzellige Lungenkarzinom

Primäres kleinzelliges Lungenkarzinom

Kolorektales Karzinom

Hamartom

? Was ist der Vorteil einer chirurgischen pulmonalen Metastasektomie?

Eine mögliche Verlängerung des Gesamtüberlebens

Eine nach den Ergebnissen von Phase-3-Studien zu erwartende Verbesserung des Gesamtüberlebens von im Median 5,4 Monaten

Ein Vorteil einer chirurgischen Metastasektomie wurde bisher nicht beschrieben.

Eine Verbesserung der Ansprechraten durch die nachfolgende Chemotherapie.

Eine Verbesserung der Lungenfunktion, da sich das pulmonale Restgewebe besser ausdehnen kann.
Ein 50-jähriger Patient mit fortbestehendem Nikotinabusus leidet an einer chronisch obstruktiven Lungenerkrankung (COPD). In der CT (Computertomographie) des Thorax zeigen zudem manche periphere Abschnitte ein Muster einer interstitiellen Pneumonie („usual interstitial pneumonia“ [UIP]). Nun wurde ein nichtkleinzelliges Lungenkarzinom im Stadium IV mit einer PD-L1-Expression (PD-L1: , programmed cell death 1 ligand 1") von $10 \%$ diagnostiziert. Der Patient leidet zudem an Schmerzen aufgrund einer recht großen Brustwandmetastase. Welche der folgenden Aussagen trifft am ehesten zu?

Eine Strahlentherapie sollte aufgrund der thorakalen Schmerzen zeitnah eingeleitet werden.

Die pulmonale Vorerkrankung stellt keine Kontraindikation gegenüber einer normal dosierten zytotoxischen Therapie in Kombination mit Checkpointinhibitoren dar.

Aufgrund der bekannten PD-L1-Expression kann auf eine molekulare Testung verzichtet werden.

Ein operatives Vorgehen ist hier vorrangig anzustreben.

$\checkmark$ Die bekannte COPD hat keine prognostische Bedeutung bei der Abschätzung der Prognose der Lungenkrebserkrankung.

? Welche Aussage zur pulmonalen Toxizität von Antitumortherapien ist nicht korrekt?

$\bigcirc$ Bei pulmonalen Nebenwirkungen ist insbesondere die Pneumonitis eine klinisch relevante Nebenwirkung.

$\bigcirc$ Eine (auch asymptomatische) medikamenteninduzierte Pneumonitis wird in der CT (Computertomographie) bei einem Großteil der medikamentös behandelten Patienten beobachtet.
Diese Fortbildung wurde von der Ärztekammer Nordrhein für das „Fortbildungszertifikat der Ärztekammer" gemäß $\S 5$ ihrer Fortbildungsordnung mit 3 Punkten (Kategorie D) anerkannt und ist damit auch für andere Ärztekammern anerkennungsfähig.

Anerkennung in Österreich: Für das DiplomFortbildungs-Programm (DFP) werden die von deutschen Landesärztekammern anerkannten Fortbildungspunkte aufgrund der Gleichwertig- keit im gleichen Umfang als DFP-Punkte anerkannt (\$ 14, Abschnitt 1, Verordnung über ärztliche Fortbildung, Österreichische Ärztekammer (ÖÄK) 2013).

\section{Hinweise zur Teilnahme:}

- Die Teilnahme an dem zertifizierten Kurs ist nur online auf www.springermedizin.de/cme möglich.

- Der Teilnahmezeitraum beträgt 12 Monate. Den Teilnahmeschluss finden Sie online beim Kurs.
- Die Fragen und ihre zugehörigen Antwortmöglichkeiten werden online in zufälliger Reihenfolge zusammengestellt.

- Pro Frage ist jeweils nur eine Antwort zutreffend.

- Für eine erfolgreiche Teilnahme müssen $70 \%$ der Fragen richtig beantwortet werden.

- Teilnehmen können Abonnenten dieser Fachzeitschrift und e.Med-Abonnenten. 
Neben zytotoxischen Medikamenten wurde eine Pneumonitis auch für Tyrosinkinaseinhibitoren und Checkpointinhibitoren beschrieben.

$\bigcirc$ Eine häufige Strategie zur Behandlung einer medikamenteninduzierten Pneumonitis sind die Pausierung der Therapie und die Gabe von Steroiden.

$\bigcirc$ Eine medikamenteninduzierte Pneumonitis ist bei früher Diagnose häufig reversibel.

? Welche pathophysiologischen Charakteristika sind nicht bei der pulmonalarteriellen Hypertonie (PAH) und bei Tumoren beschrieben worden?

Induktion von Proliferation

Antiapoptose

Induktion von Angiogenese

$\bigcirc$ Unbegrenzte Replikation

Produktion und Freisetzung stromaler proinflammatorischer Stimuli

? Das idiopathische Pneumoniesyndrom ist eine gefürchtete Komplikation in der frühen Prä-Engraftment-Phase nach allogener Stammzelltransplantation. Wie viele der betroffenen Patienten überleben diese Komplikation?

○ $20 \%$

○ $40 \%$

○ $60 \%$

○ $80 \%$

○ $95 \%$
? Bei welcher Therapie ist die Gefahr, ein „cytokine release syndrome" zu entwickeln, am höchsten?

- CART (chimäre Antigenrezeptor-T-Zellen)

O PD-1-Inhibitoren (PD-1: „programmed death protein 1“)

$\bigcirc$ PD-L1-Inhibitoren (PD-L1: „programmed cell death 1 ligand 1“)

Cyclophosphamid

Methotrexat

? Eine pulmonale venookklusive Erkrankung ist als Nebenwirkung welcher Therapie beschrieben?

CART (chimäre Antigenrezeptor-T-Zellen)

- PD-1-Inhibitoren (PD-1: „programmed death protein 1“)

$\bigcirc$ PD-L1-Inhibitoren (PD-L1: „programmed cell death 1 ligand 1“)

Cyclophosphamid

- Methotrexat

? Eine 64-jährige Patientin (40 Patientenjahre [py]) mit kleinzelligem Adenokarzinom im Stadium III soll eine Radiochemotherapie bekommen. Wie hoch ist ihr Risiko, eine Strahlenpneumonitis zu entwickeln?

- Um $5 \%$

○ Um $15 \%$

○ Um $25 \%$

○ Um $35 \%$

○ Um $45 \%$

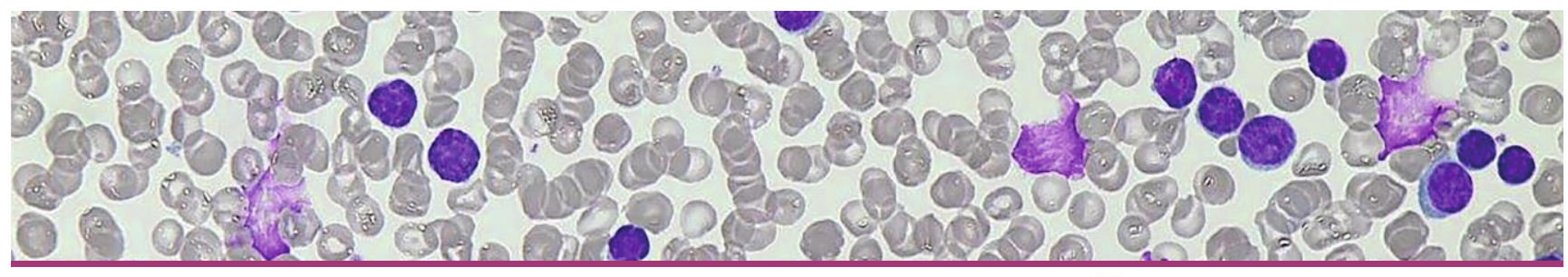

Der neue Kurs: CLL DGFM M

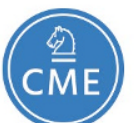

$\checkmark$ Leitlinienorientiert und fallbasiert

$\checkmark$ Optimiert für Smartphones

$\checkmark$ Zertifiziert mit 3 Punkten

Diesen CME-Kurs finden Sie auf " DGIM-eAkademie.de

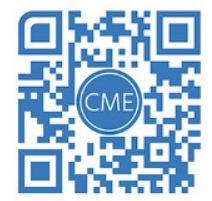

Das Fortbildungs-Portal der DGIM: Kostenfrei für alle Mitglieder und e.Med-Abonnenten 
Hier steht eine Anzeige.

\section{Springer}

